\title{
Estimation of Bending Resistance of Ionic Polymer Metal Composite (IPMC) Actuator Following Variable Parameters Pseudo-Rigid Body Model
}

\author{
Dibakar Bandopadhya ${ }^{1}$ and James Njuguna ${ }^{2}$ \\ ${ }^{1}$ Department of Mechanical Engineering, Indian Institute of Technology Guwahati \\ Guwahati, India \\ ${ }^{2}$ Centre of Automotive Technology, Department of Sustainable Systems, Cranfield University, \\ Bedfordshire, MK43 0AL, UK
}

\begin{abstract}
In this article bending resistance of IPMC has been estimated following a newly proposed Variable Parameters Pseudo-rigid Body model. First an experiment is conducted to study the voltage verses bending characteristics of IPMC and based on the experimental data the IPMC has been modeled through the proposed technique. Simulation has been performed and estimation of bending resistance has been made based on experiment results. It is observed that bending resistance of IPMC increases with input voltages although the changes remain insignificant after certain range.
\end{abstract}

Key words: Ionic Polymer Metal Composite, Electro-Active Polymer, Pseudo-rigid Body Model, Variable Length Rocker, Shape Memory Materials, Elastic Properties

\section{Introduction}

In the quest for advanced material Ionic Polymer Metal Composite (IPMC) is an Electro-active Polymer (EAP) that is gaining great importance as sensor and actuator. The advantages of IPMC in requiring low activation voltage and the induced large bending strain i.e. capability of transforming electrical energy into mechanical energy and vice versa, make it suitable for various potential applications like in robotics, aerospace and biomimetics [1-3]. It consists of thin polyelectrolyte membrane and a type of noble metal, such as gold and platinum, chemically plated on both sides of the membrane. An IPMC can undergo fast and large bending motion when a low electric potential is applied to its. Conversely, an IPMC can generate a measurable electric potential when it is subjected to a sudden bending. Thus IPMC can serve as both actuator and sensor. An IPMC may offers advantages, such as compliant, lightweight, low voltage operation and capability of working in aqueous environments. These properties make it promising for numerous applications in biomedical, naval, robotic and microelectromechanical system (MEMS) engineering [4-5]. 
In the present study IPMC has been modeled following variable parameters pseudo-rigid body modeling technique for estimation of bending resistance. The pseudo-rigid-body modeling technique is employed to simplify the analysis and design of compliant mechanisms. Such model approximates a compliant mechanism by an appropriate, equivalent rigid-body mechanism. The model is based on the fixpin support type of cantilever mode with end-moment loading [6-7] and its derivation is explained in detail. Based on the experimentally obtained deflection data and the bending moment generated, IPMC has been modeled to obtain the variable spring constants, characteristic radius factors, pseudo-rigid body angles. The expressions cover practical range of applications using a single equation for each variable. The accuracy of the model is excellent and could be compared with the fixed radius pseudo-rigid body model of IPMC.

Simulations have been performed based on the experimental data and demonstration has been made to estimate the bending resistance of IPMC for various input voltages and the subsequent tip position during bending. The proposed model also validates the results pertaining to the material properties, assumptions taken with the experimental results and the bending moment generated due to input voltage.

\section{Pseudo-Rigid Body Model of IPMC}

Pseudo-rigid body modeling technique is used to model the compliant/partially compliant mechanism by an appropriate equivalent rigid-body mechanism [8]. Pseudo-rigid body model of IPMC is dominated by the bending moment generated due to input voltage. It is observed nonlinear bending characteristics of IPMC under potential gradient. Because of this, experimental results are obtained and subsequently IPMC has been modeled using the proposed method to estimate it's bending resistance. An analogy has been drawn that the bending phenomena is equivalent to the same amount of tip deflection that is caused by external bending moment $M=\frac{E I}{R}$ acting at the tip of IPMC. Where, EI is the flexural rigidity of IPMC and R is the radius of curvature. This concept is employed here to model the IPMC for each bending moment generated for each input voltage. Voltage is applied at one end and subsequently end point deflection has been obtained. The pseudo-rigid body model of the deflected IPMC with various parameters is shown in Figure 1. Here, the IPMC is replaced by a link with a characteristic pivot located at a length $\gamma \boldsymbol{l}$ from the free end of the IPMC. It is assumed that a circular path can be accurately modeled by two rigid links that are connected at a pivot along the IPMC strip. A torsional spring at the pivot represents 
the IPMC strip's resistance to bending. Therefore, our main objective is to establish relationship and to estimate the variation of spring constant with input voltages. The location of this pseudo-rigid-body characteristic pivot is measured from the IPMC tip end as a fraction of the IPMC length, where the fractional distance is $\gamma \boldsymbol{l}$ and $\gamma$ is the characteristic radius factor. The product $\gamma \boldsymbol{l}$, the characteristic radius, is the radius of the circular deflection path traversed by the end of the pseudo-rigid body link. It is also the length of the pseudo-rigid body link. This section will address the newly proposed variable parameters pseudo-rigid body model for estimation of bending resistance. Improvement of the model is based on finding distributed force, variable parameters such as stiffness, pseudo-rigid body angle and characteristic radius factor. Based on these parameters IPMC bending model has been studied and subsequently bending resistance of IPMC has been assessed for various input voltages. Figure 1 shows the correlation among various variables of deflected IPMC with its pseudo-rigid body model.

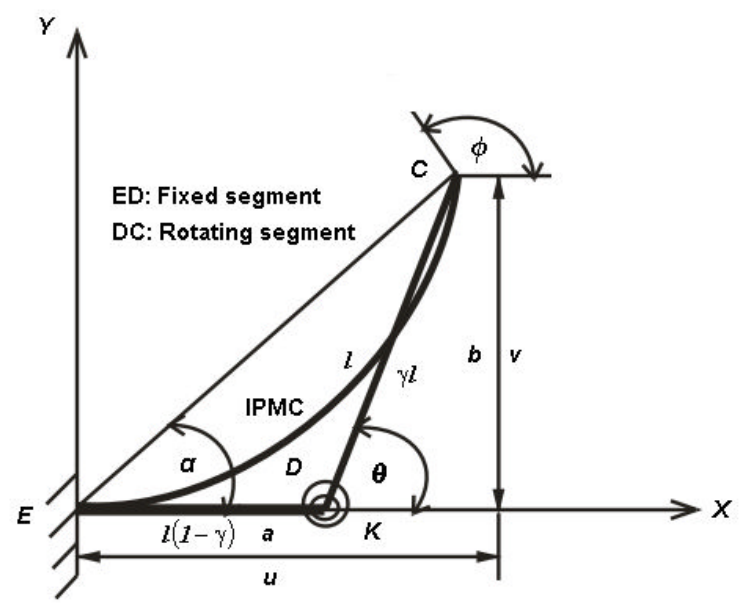

Figure 1.

Using figure 1, one can establish the following relationship [6]:

$u=\frac{E I}{M} \sin \phi, \quad v=\frac{E I}{M}(1-\cos \phi)$

$\tan \alpha=\frac{v}{u}$

$a=l\left(1-\gamma_{v}\right)+\gamma_{v} l \cos \theta_{v}, \quad b=\gamma_{v} l \sin \theta_{v}$

where, $\gamma_{v}$ is the variable characteristic radius factor and $\theta_{v}$ is the pseudo-rigid body angle. Further,

$E D+D C=l \Rightarrow x+\sqrt{(u-x)^{2}+v^{2}}=l$

where, $\boldsymbol{E D}=\boldsymbol{x}$, on simplification equation (4) leads to 
$x=\frac{u^{2}+v^{2}-l^{2}}{2(u-l)}$

Therefore, using equation (6), one can establish the relationship for variable characteristic radius factor as:

$l\left(1-\gamma_{v}\right)=\frac{u^{2}+v^{2}-l^{2}}{2(u-l)} \quad \Rightarrow \gamma_{v}=1-\left[\frac{u^{2}+v^{2}-l^{2}}{2 l(u-l)}\right]$

Subsequently, pseudo-rigid body angle is obtained as:

$\theta_{v}=\sin ^{-1}\left(\frac{b}{\gamma_{v} l}\right)$

Therefore, bending moment generated at the tip of the IPMC is obtained as:

$M_{v}=P\left(a-l\left(1-\gamma_{v}\right)\right)=\frac{E I}{R}$

Hence, the variable spring constant for the IPMC is obtained as:

$\boldsymbol{K}_{v}=\frac{\boldsymbol{M}_{v}}{\theta_{v}}$

This spring constant of IPMC measures the bending resistance under potential gradient.

\section{Results and Discussions}

Based on the experimentally obtained deflection data of an IPMC actuator $\left(40 \times 5 \times 1 \mathrm{~mm}^{3}\right)$ as shown in

Table 1, numerical simulation has been done and the variable parameters are obtained and plotted. The tip deflections data are taken for 30 seconds for each input voltage. All programs are developed in MATLAB. A single patch of IPMC of Elastic Modulus $1.2 \mathrm{GPa}$ and mass $0.67 \mathrm{gm}$ has been analyzed and the bending resistance of IPMC has been assessed in terms of spring constants. Figure 2 shows the variation of characteristic of radius factor for different input voltages while Figure 3 shows the change in pseudo-rigid body angles. Figure 4 and 5 are showing the change in tip position of IPMC and the corresponding change in spring constant for each input voltage. Figures 2-4 clearly demonstrated the nonlinear variation of parameters with input voltages. 
Table 1.

\begin{tabular}{|c|c|c|c|c|c|c|c|c|c|c|}
\hline $\begin{array}{c}\text { Input voltage (V) } \\
\begin{array}{c}\text { X-coordinate (u) } \\
(\mathrm{mm})\end{array}\end{array}$ & 0.5 & 1 & 1.5 & 2 & 2.5 & 3 & 3.5 & 4 & 4.5 & 5 \\
\hline $\begin{array}{c}\text { Y-coordinate (v) } \\
(\mathrm{mm})\end{array}$ & 3 & 6.5 & 9.5 & 12.5 & 15.5 & 18 & 20.5 & 23 & 25 & 26.5 \\
\hline $\begin{array}{c}\text { Radius of } \\
\text { curvature (R) } \\
(\mathrm{mm})\end{array}$ & 213.3 & 106.6 & 71.1 & 53.3 & 42.6 & 35.5 & 30.5 & 26.6 & 23.7 & 21.3 \\
\hline
\end{tabular}

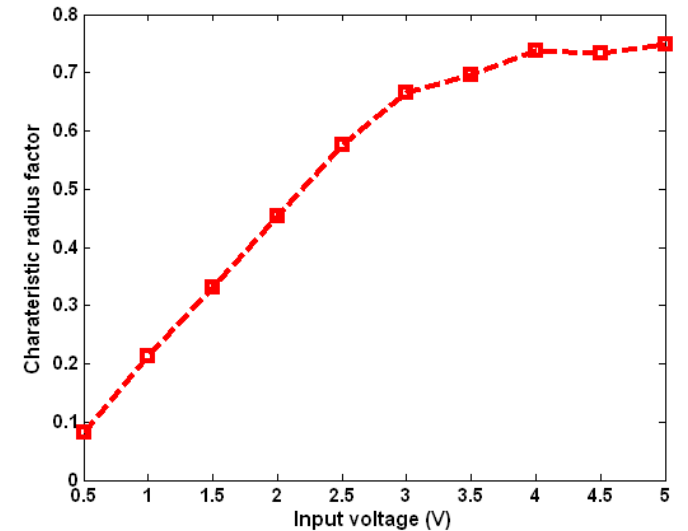

Figure 2.

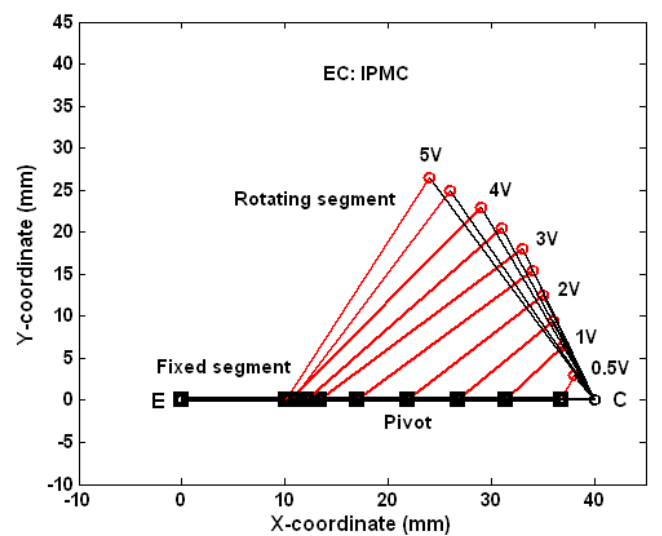

Figure 4.

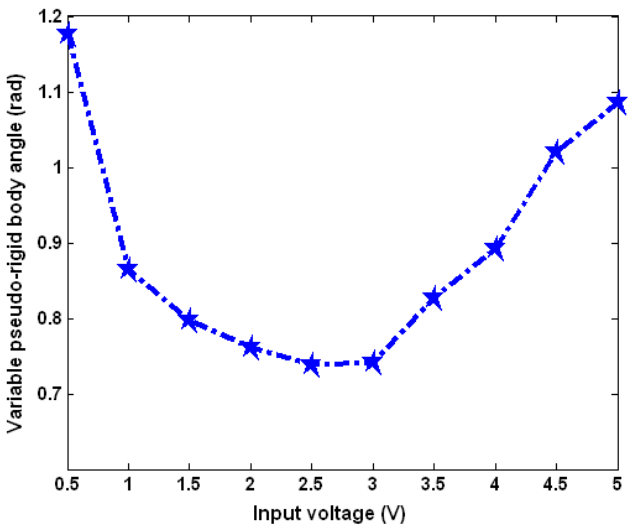

Figure 3.

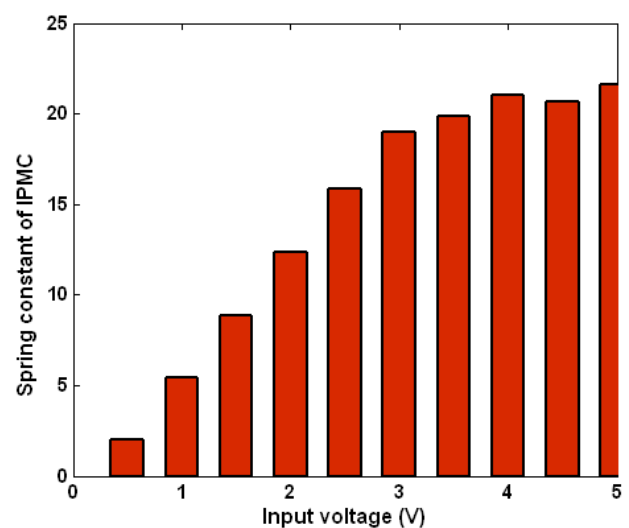

Figure 5.

\section{Conclusion}

In this article a newly proposed variable parameters pseudo-rigid body model of IPMC actuator has been studied for bending resistance estimation with input voltages. The model is based on the experimental 
deflection data. It has been demonstrated how the bending resistance of IPMC changes with change in tip position. The model also clearly demonstrates the variation of torsional spring constant and their characteristics pivot locations.

\section{References}

[1] Shahinpoor M, Kim KJ, Smart Mater Struct 2001;10:819-33.

[2] Kim KJ, Shahinpoor M, Smart Mater Struct 2003;12: 65-9.

[3] Shahinpoor M, Kim KJ, Smart Mater Struct 2004;13:1362-88.

[4] Shahinpoor M, Kim KJ Smart Mater Struct 2005;14:197-214.

[5] Nemat-Nasser S, Li JY, J Appl Phys 2000;87: 3321-31.

[6] Bandopadhya D, Dutta A, Bhattacharya B, J Intelligent Mater Syms Struct, 2008, doi:10.1177/1045389X08088784.

[7] Bandopadhya D, J Reinfor Plast Compos, 2008; doi: 10.1177/0731684407086998

[8] Howell L, Midha A, Norton TW, ASME J Mech Des 1996;118:125-131.

\section{List of Figures and Tables}

Figure 1. Correlation between deflected IPMC with its Pseudo-rigid body model.

Figure 2. Variation of characteristic radius factor of IPMC with input voltage.

Figure 3. Changes of pseudo-rigid body angle of IPMC with input voltage.

Figure 4. Change in tip position and the characteristic pivot of IPMC following the variable parameters pseudo-rigid body model.

Figure 5. Variation of spring constant of IPMC with input voltage.

Table 1. Experimentally obtained tip position of deflected IPMC. 